\title{
STUDY OF ABSORPTION IN CARBON NANOTUBE COMPOSITES FROM 1HZ TO 40GHZ
}

\author{
Aline Emplit and Isabelle Huynen \\ Institute of Communication Technologies, Electronics and Applied Mathematics \\ (ICTEAM), Université catholique de Louvain, Louvain-la-Neuve, Belgium
}

\begin{abstract}
Absorption performances in High Density Polyethylene (HDPE) and polycarbonate (PC) polymer matrices containing various loads of carbon nanotubes were analysed. It depends on electrical conductivity, dielectric constant and thickness of the polymer composites. These parameters can be easily controlled. Significant absorption, which can reach between 60 and 90\%, hence occurs at particular combinations of these last parameters (in a frequency range from $1 \mathrm{~Hz}$ to $\mathrm{GHz}$ ). These new results are really useful in various applications, and are considered in low scale systems as a major technological solution against electromagnetic interferences.
\end{abstract}

\section{KEYWORDS}

Microwave absorbers, electromagnetic interferences, nanocomposites, carbon nanotubes

\section{INTRODUCTION}

Reduction of electromagnetic pollution and electromagnetic interferences is a major technological issue considering the widespread use of wireless applications. The protection of living cells or the preservation of the operation of sensitive electronic devices constitute prominent examples. In most applications the unwanted radiation is either confined at the source or the device is shielded by a metallic enclosure that reflects the incident radiation. Most of the time this approach provides cheap and effective solutions. But for some applications shielding based on true absorption of the EM wave with no reflection and no transmission is needed or at least preferred. Various topologies of absorber have been developed [1] : Salisbury screens, Dallenbach and Jauman layers. They however do not operate on a wide range of frequencies. One example of application is radar stealth, which consists in reducing the detectability of a target by cancelling reflection of a radar signal incident to the surface. Usually radar absorbing materials are made of thick foam designed for wideband absorption. Alternatively, thin multilayered coatings can be used but with operation usually restricted to a narrow frequency band, except for carbon nanotube (CNT) nanocomposites [2-3]. Another approach consists in Frequency-Selective Surfaces (FSS) that enable to achieve nearly perfect absorption, however in a narrow frequency band since planar resonators are used to achieve absorption at resonance [4-5]. A further development combines FFS structures with nanocomposite lossy slabs [6-7]. On the other hand, there is few literature about composites for absorption below $1 \mathrm{GHz}$. EMI shielding was observed around $200 \mathrm{MHz}$ using carbon nanotubes in poly-methyl-metacrylate [8] and polyacrylate [9]. Up to 30 and $25 \mathrm{~dB}$ of shielding were obtained respectively. However these performances result from a combination of reflection and absorption rather than pure absorption. Carbon nanotubes are also efficient for absorption above $1 \mathrm{GHz}$. Indeed their high aspect ratio promotes the formation of a conductive path for a low content [10]. Mechanical behaviour of absorbers is also of prime interest in airborne applications. Therefore some solutions were investigated combining CNT composite and honeycomb metallic matrix in order to improve resistance to shocks [11]. 
In this paper we perform a thorough study of the absorption performances of nanocomposites polymer slabs including carbon nanotubes [2-3]. Compared to the state-of-the-art, it involves both low and high frequencies for the same composites, which is rather unique to our best knowledge. Section II reminds on the electromagnetic formulation of absorption performances depending on thickness of the slab absorber and its conductivity. Sections III and IV briefly describe the preparation of the composites and their structural morphology. Section V deals with electrical properties, and section VI with frequency response of the absorption.

\section{THEORETICAL FORMULATION OF ELECTROMAGNETIC ABSORPTION}

Slab absorption is the fraction of power which is neither reflected nor transmitted, and depends on several parameters such as frequency (f), thickness (d), dielectric constant $\left(\varepsilon_{\mathrm{r}}\right)$ and conductivity $\sigma$. The reflection coefficient $\Gamma$ at input interface of slab and the transmission factor $T_{\mathrm{f}}$ over slab thickness $d$ follows the relations:

$\Gamma=\frac{\sqrt{\varepsilon_{e f f}}-1}{\sqrt{\varepsilon_{e f f}}+1}$

$T_{f}=e^{-\gamma d}$

$\gamma=\alpha+j \beta=j \varpi \frac{\sqrt{\varepsilon_{e f f}}}{c_{o}}$

Where $\omega=2 \pi \mathrm{f}$ and $\varepsilon_{\mathrm{ff}}$ is the effective permittity:

$\varepsilon_{e f f}=\varepsilon_{r}+\frac{\sigma}{j \varpi \varepsilon_{o}}$

With $\varepsilon_{\mathrm{r}}$ the relative dielectric constant, while in (3) $\alpha$ is the attenuation coefficient and $\beta$ the propagation constant.

Reflection and transmission ( $\mathrm{R}$ and $\mathrm{T})[12]$ are:

$R=\left\lfloor\frac{\Gamma\left(T_{f}^{2}-1\right)}{1-\Gamma^{2} T_{f}^{2}}\right\rfloor$

$T=\left\lfloor\frac{T_{f}\left(1-\Gamma^{2}\right)}{1-\Gamma^{2} T_{f}^{2}}\right\rfloor$

The absorption A can be written as

$$
\begin{aligned}
& \mathrm{A}=1-\mathrm{P}_{\mathrm{r}}-\mathrm{P}_{\mathrm{t}}=1-R-T \\
& =1-\left|\frac{\Gamma\left(T_{f}^{2}-1\right)}{1-\Gamma^{2} T_{f}^{2}}\right|^{2}-\left|\frac{T_{f}\left(1-\Gamma^{2}\right)}{1-\Gamma^{2} T_{f}^{2}}\right|^{2}
\end{aligned}
$$

Where $\mathrm{P}$ is the incident power, $\mathrm{P}_{\mathrm{r}}$ the reflected power and $\mathrm{P}_{\mathrm{t}}$ the transmitted power, while $\mathrm{A}$ is ranging between 0 and 1 . 


\section{Preparation OF NANOCOMPOSITES}

Two materials (matrix) were used: PC and HDPE. The interest was to compare two kinds of behaviour, each one associated to a different quality of nanoparticle dispersion.

A twin-screw mini-compouder was used to compound the HDPE composites. For all melt blending experiments, the conditions were as following : $250 \mathrm{rpm}, 10 \mathrm{~min}$ compounding. The processing temperature was $220^{\circ} \mathrm{C}$. For further characterization of the electrical properties the samples were compression-molded at $190^{\circ} \mathrm{C}$ for $1 \mathrm{~min}, 10 \mathrm{~min}$ or $20 \mathrm{~min}$ respectively in a Fontjne press followed by quick cooling. The HDPE nanocomposite samples were provided by Nanocyl company and contain 1 to 15 wt\% CNT. Commercial CNT NC7000 were used. Compounds were processed in a commercial extruder. For further characterization of electrical properties, samples were injected-molded in an Engel, Victory injection-molding machine. This technique is commonly used by companies. This implies that results are interesting for research and industry as well.

PC nanocomposites were fabricated by melt-blending in an extruder (Brabender extruder) at $270^{\circ} \mathrm{C}$ for $5 \mathrm{~min}$; and were composed of PC Macrolon 2805 (Bayer) with a fraction of CNT (Nanocyl NC7000). The compounds were compression-molded at $290^{\circ} \mathrm{C}$ for $2 \mathrm{~min}$ after extrusion, under 15 tons.

\section{MORPHOLOGY OF NANOCOMPOSITES}

CNT dispersion is homogeneous in PC composites (Fig. 1) and not homogenous in HDPE composites (Fig. 2). Entangled CNT in network form agglomerates. Fig. 2 shows the CNT network in compound with $5 \%$ of particle concentration.

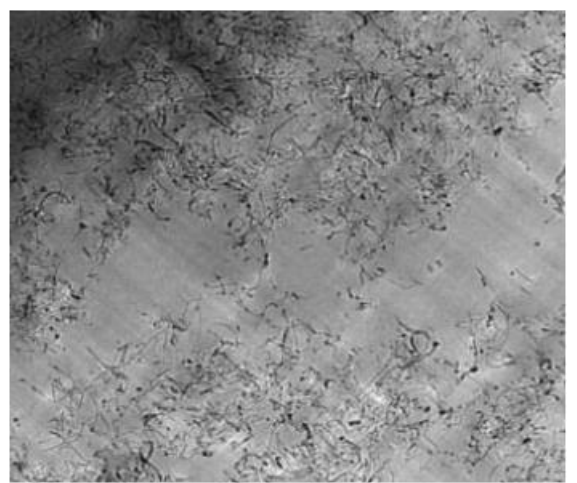

Fig. 1. TEM observation of PC nanocomposite loaded with carbon nanotubes (from [6]).

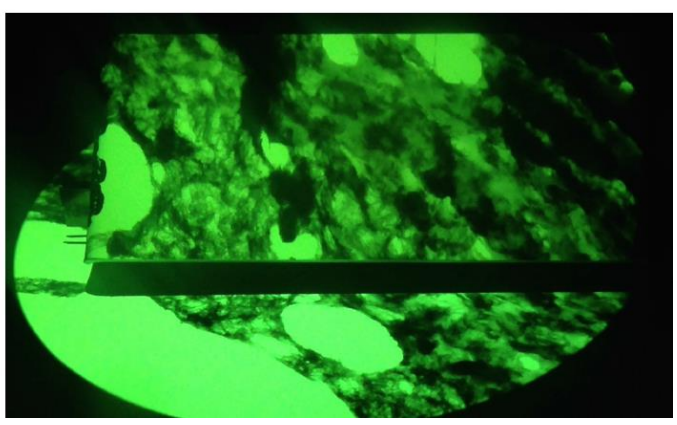

Fig. 2. TEM image of HDPE nanocomposite loaded with carbon nanotubes $\left(\operatorname{area}=1 \mathrm{~mm}^{2}\right)$. 


\section{ELECTRICAL PROPERTIES}

Low frequency measurement (from $0.01 \mathrm{~Hz}$ to $1 \mathrm{MHz}$ ) was performed using a Novocontrol AlphaA High Performance Frequency Analyser connected to an Agilent Dielectric Test Fixture. Resistance (R) and capacitance (C) were measured between two electrodes which stack the sample on each edge, with classical RC parallel equivalent circuit as model for the specimen. DC measurements used a 4-probes system connected to a Keitley source.

The continuity and the respect of the power law are also checked here by additional ' $\mathrm{GHz}$ measurements'. High frequency measurements use a microstrip transmission line method described in [11], with a Vector-Network Analyser Model Wiltron 360.

Compound based on PC matrix was largely studied up to now in GHz frequency range. New sub$100 \mathrm{MHz}$ results (obtained here) are hence compared to these literature results. The electrical properties are measured for a volume $\left(\right.$ several $\mathrm{mm}^{3}$ ) enclosed between two electrodes. The preparation step included a metallization of electrodes on each side of the samples, removing roughness contribution (to accuracy limit) during electrical measurement.

Best results of sub- $100 \mathrm{MHz}$ absorption were obtained in compounds with a lower quality of particle distribution such as compounds based on HDPE matrix. Indeed, higher CNT concentration was needed to reach high sub-100MHz absorption, but reminding this absorption behaviour was really complex and depends on different other parameters.

\subsection{AC conductivity}

Fig. 3 and 4 show the AC conductivity, respectively for PC and HDPE compounds. AC conductivity of PC nanocomposites are measured by two different techniques and the values are extracted from measurement by different models. The curves fit a named power law behaviour, used to explain the electrical conductivity of these kinds of materials [4,13]. This confirms a good continuity of the AC conductivity, depending on the frequency: between 10 and $40 \mathrm{GHz}$ the curves are almost parallel, and below $1 \mathrm{MHz}$ some curves diverge to reach a plateau. In $\mathrm{GHz}$ frequency, the values around 1 are in good agreement with previous measurements results [13] while in sub-100MHz range, a slightly different behaviour exists due to improvement of the measurement quality (by removing the roughness impact on measurement): the 'conductor' has a higher conductivity (difference around 2 to 3 orders of magnitude). DC conductivities around 10${ }^{12}, 5.10^{-5}, 10^{-4}, 0.5$ and $10 \mathrm{~S} / \mathrm{m}$ have already been obtained respectively at $0,0.5,1,1.5$, and $2 \mathrm{wt} \%$ of CNT in PC compounds [9] (four probe measurement technique), validating AC conductivities values described here.

An insulating electrical behaviour was observed at concentrations below 3\% in HDPE compounds (Fig. 4). Electrical conductivity was frequency-independent at concentrations higher than $5 \%$, and reach 0.01 to $100 \mathrm{~S} / \mathrm{m}$ at concentrations from 5 to $15 \mathrm{wt} \%$.

HDPE compounds had higher conductivities, around $1 \mathrm{~S} / \mathrm{m}$ at $10 \mathrm{wt} \%$ load of CNT particles. Low dispersion of particles resulted in local network of entangled CNT. Observed values are hence very high, quite similar to the values obtained in $\mathrm{GHz}$ frequency range $[4,13]$.

Power law dependence was observed at concentrations close to percolation threshold. Electrical conductivity mechanism was based on charge hopping of carriers between CNT inside the network. Some barriers exist between neighbouring CNT, limiting the electrical conductivity. CNT network and dispersion (agglomerates/clusters or isolated) modifies the interparticle distance: the potential barrier between neighbouring CNT changes as a function of frequency and 
hopping conditions are modified. This can increase or decrease the load of conductive filler necessary at percolation.

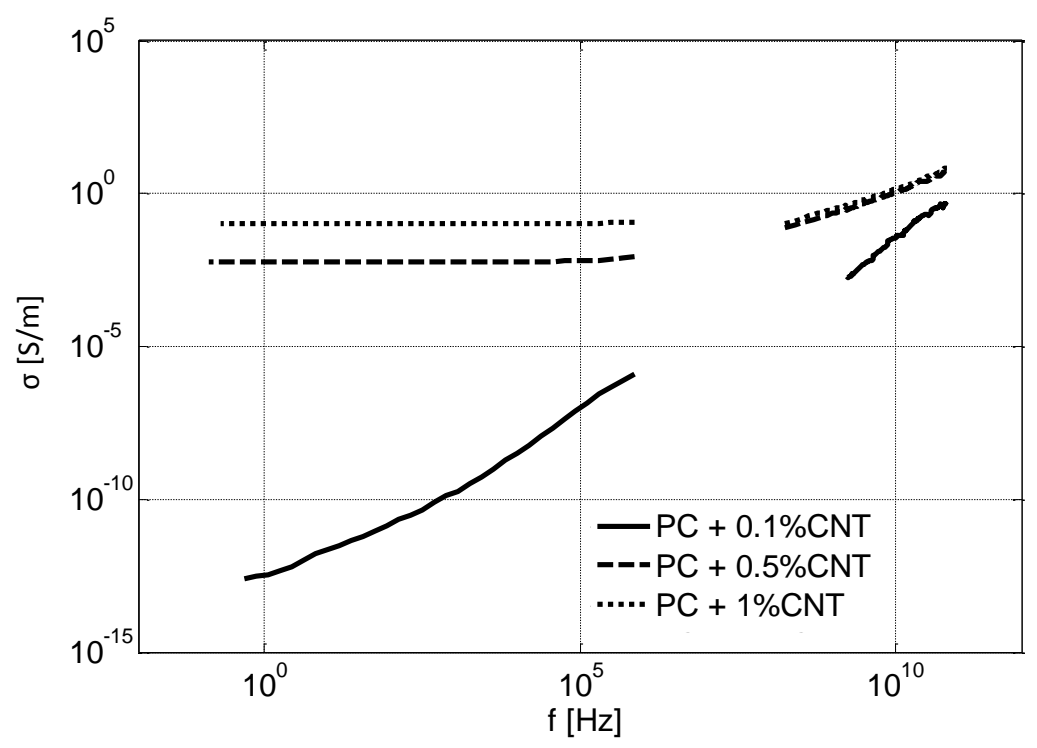

Fig. 3. Conductivity of PC samples as a function of loading charge and frequency.

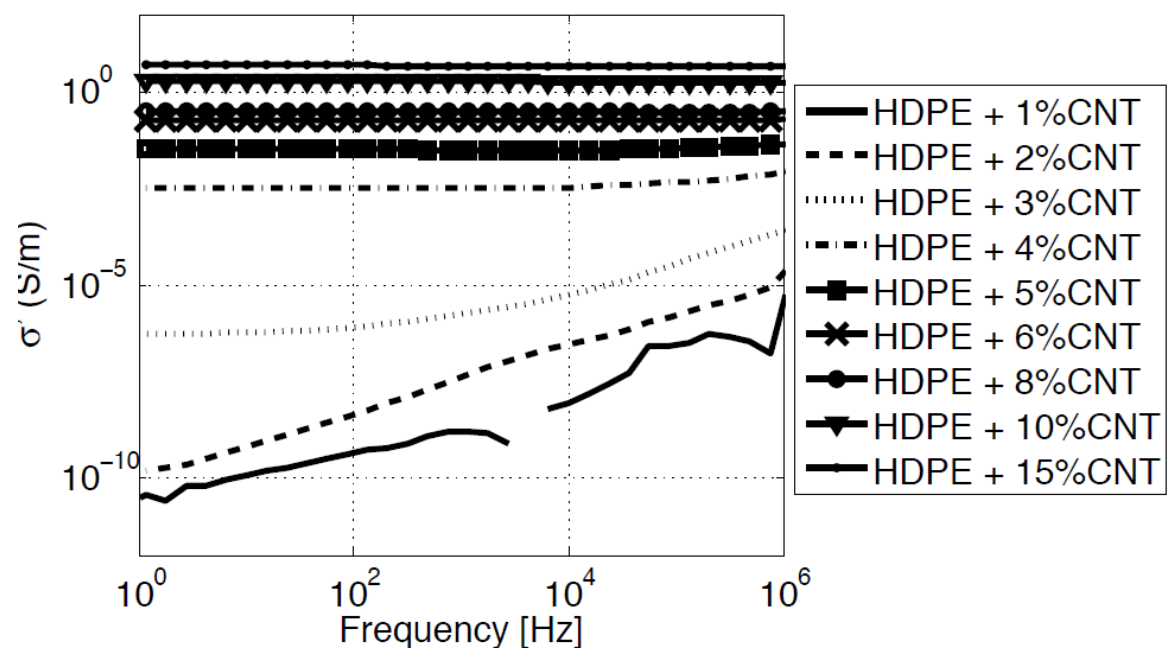

Fig. 4. Conductivity of HDPE samples for loading charge of CNT as a function of frequency.

\subsection{Dielectric constant}

In Fig. 5, dielectric constants of PC compounds are in same order of magnitude and close to measurement in literature [13].

Dielectric constant of HDPE composites shown in Fig. 6 was around 1000 for samples containing more than 4wt \% CNT. Dielectric constant was around 3, 10 and between 10 and 100 respectively for $\mathrm{HDPE}+1 \%, 2$ and $3 \%$ of CNT.

Dielectric constant is highly influenced by molecular relaxation phenomenon inside composite. An applied stimulus (electric field) results in a polarization response of material. The value drops from a constant value at low frequency to a lower value at high frequencies. This behaviour is 
explained in literature: for non ohmic behaviour or when a polarization effect occurs, the real part of permittivity increases with decreasing frequency. This phenomenon is also influenced by the loading charge. For high loading charges, a high dielectric constant is reached compared to lower values measured at low loading charges. The relaxation frequency depends on the loading: higher the load, higher the frequency.

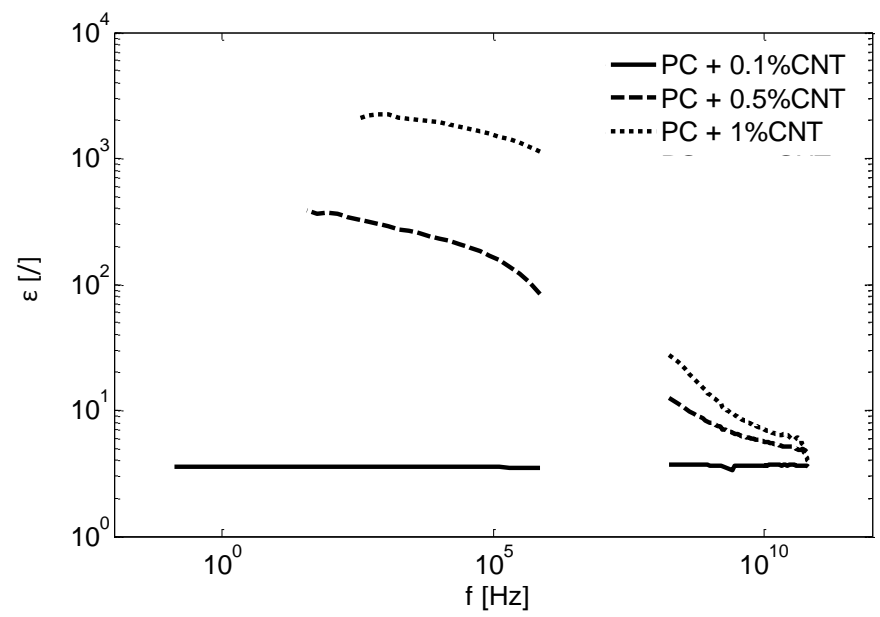

Fig. 5. Dielectric constant of PC samples as a function of loading charge of CNT and function of frequency

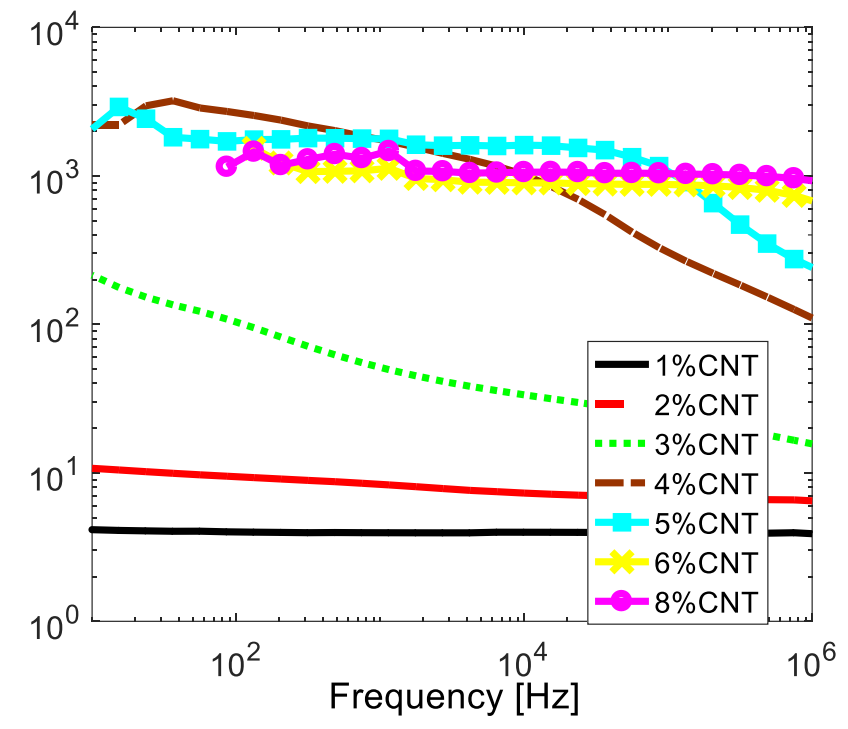

Fig. 6. Dielectric constant of HDPE samples as a function of loading charge of CNT and frequency (from [13]).

\section{FREQUENCY RESPONSE OF ABSORPTION}

\subsection{Percolation threshold and electromagnetic behaviour}

Adding metallic CNT increases both electrical conductivity and dielectric constant of the compounds. The impact on the dielectric constant is less pronounced than for the conductivity: this implies more influence on transmission than on reflection. Reflection increases as a function 
of loading charge, while transmission decreases progressively. The higher the CNT loading, the more the material becomes opaque to the incident wave.

Optimal absorption is obtained at low dielectric constant and a high electrical conductivity, which implies a low reflection and a low transmission.

In this section absorption of PC and HDPE nanocomposites are calculated using formulas (1-8) yielding absorption using measured values of dielectric constant and conductivity shown in Fig. 5 and 6.

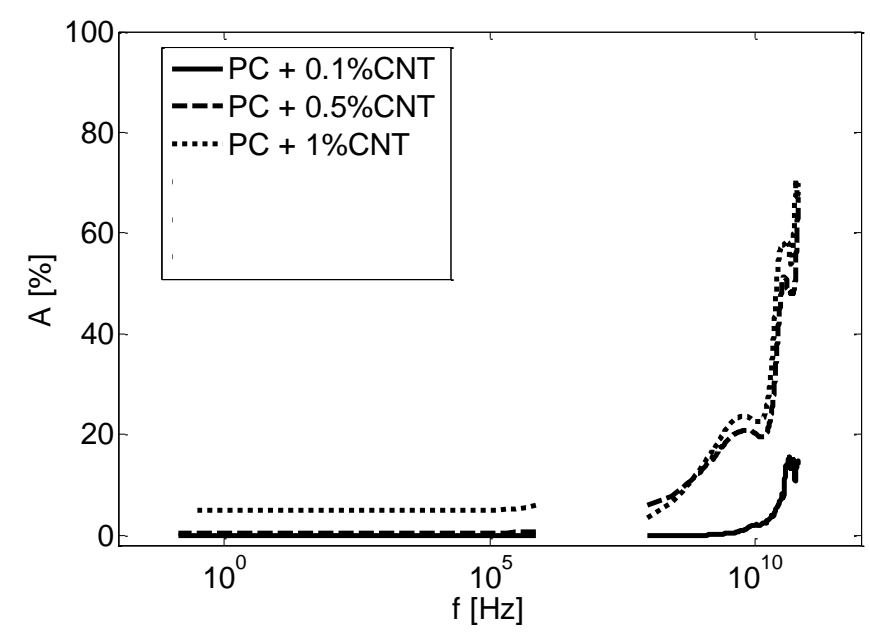

Fig. 7. Conductivity of PC samples as a function of CNT concentration as function of frequency (considered thickness: $2 \mathrm{~mm}$ ).

Absorption of PC compounds on a large frequency range, in Fig 7, increases progressively as a function of loading charge. The curves extracted from two measurement techniques followed a nice continuous behaviour. Below percolation threshold, both electrical conductivity and dielectric constant were low. Above this threshold, the values increase and hence result in an increase of absorption. Below $100 \mathrm{MHz}$, conductivity (showing an increase of 10 orders of magnitude) was the main factor increasing absorption, but was limited by a strong increase of dielectric constant ( 3 orders of magnitude). Absorption remained higher in $\mathrm{GHz}$ frequency range $[4,14]$ : conductivity was still high but dielectric constant falls to a lower value.

\subsection{HDPE compounds - below 100MHz}

Let us compare HDPE compounds (Figs. 8-10). Reflection increased and transmission decreased at increasing loading, but the behavior was not exactly opposite: a difference occurs due to absorption.

First reduction of transmission occurs at percolation threshold (around 3wt $\% \mathrm{CNT}$ ), but reflection increases only for more than $5 \mathrm{wt} \%$ concentration. Transmission ranged from $100 \%$ to $80 \%$ at 1 to $8 \mathrm{wt} \% \mathrm{CNT}$; and reflection is around 10 and $42 \%$ respectively at 10 and $15 \mathrm{wt} \% \mathrm{CNT}$.

Best absorption is obtained at $10 \mathrm{wt} \%$ concentration, hence not at higher concentration (15wt \%). However, transmission is lower at $15 \mathrm{wt} \% \mathrm{CNT}$, due to a significant reflection added to absorption. This transmission and absorption behaviors have been largely described in literature for different compounds in $\mathrm{GHz}$ frequency range [2,14,15]: absorption can reach $90 \%$ above $1 \mathrm{GHz}$ but dramatically decreases under $1 \mathrm{GHz}$. Simulated absorption index has been estimated to 
International Journal Of Microwave Engineering (JMICRO) Vol.2, No.1, January 2017

$50 \%$ under $10 \mathrm{GHz}$ in $[6,7]$ for a nanocomposite slab with a dielectric constant of 3 , an electrical conductivity of $1 \mathrm{~S} / \mathrm{m}$ and a thickness of $10 \mathrm{~mm}$.

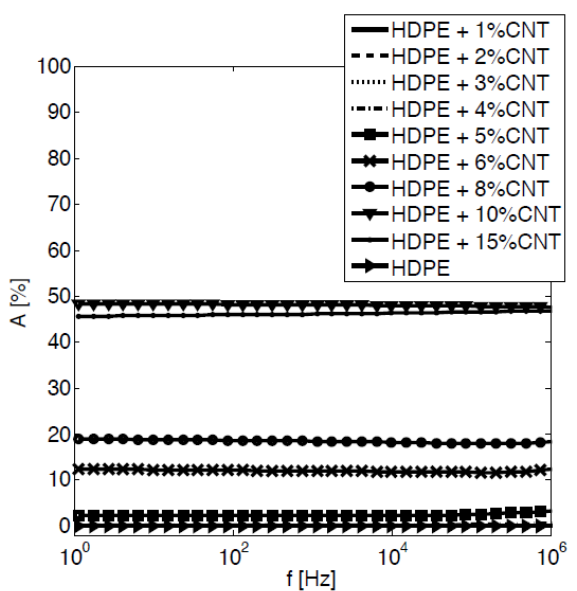

Fig.8. Absorption vs frequency of HDPE samples.

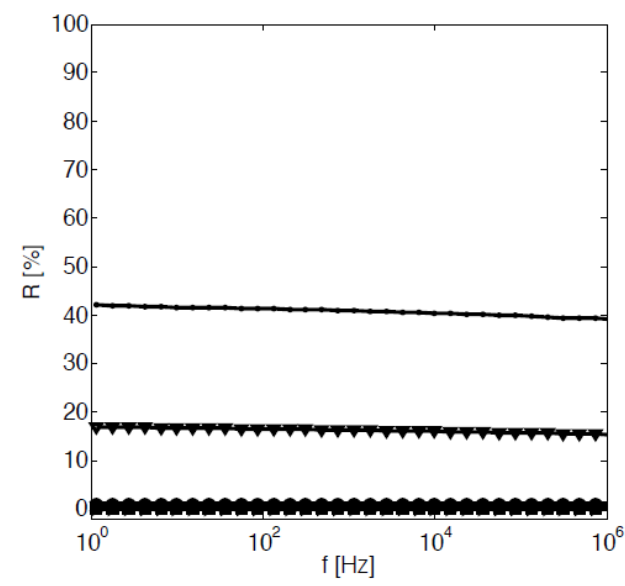

Fig. 9. Reflection vs frequency of HDPE samples.

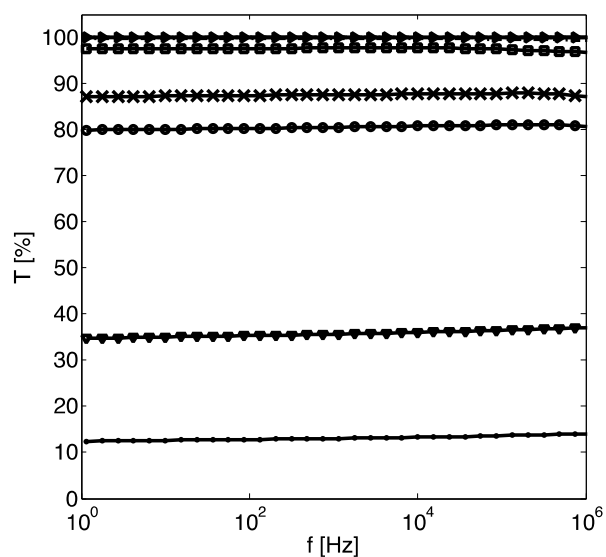

Fig. 10. Transmission vs frequency of HDPE samples. 


\subsection{Thickness of sample and electromagnetic behaviour}

Thickness (d) impacts the transmission factor (2), and hence modifies both reflection and transmission. Since $\varepsilon_{\mathrm{ff}}$ is complex, transmission factor includes an attenuation term responsible for absorption and a phase shift. Thickness has an impact even if the slab is very small compared to the wavelength. Optimal absorption follows a specific behaviour as a function of thickness, depending on both reflection and transmission behaviours.

\subsection{1 $1 \mathrm{~Hz}-1 \mathrm{MHz}$ frequency range}

Best absorption by PC samples (Fig. 11) is obtained at $1 \mathrm{wt} \%$ CNT. Transmission in Fig. 13 decreased for matrix containing more than $0.5 \mathrm{wt} \%$, and reflection in Fig. 12 increases at higher CNT loading than $1 \mathrm{wt} \%$. The curves reach respectively $100 \%$ and $0 \%$ at different thicknesses. The differences between the described impacts have a direct contribution on the absorption.

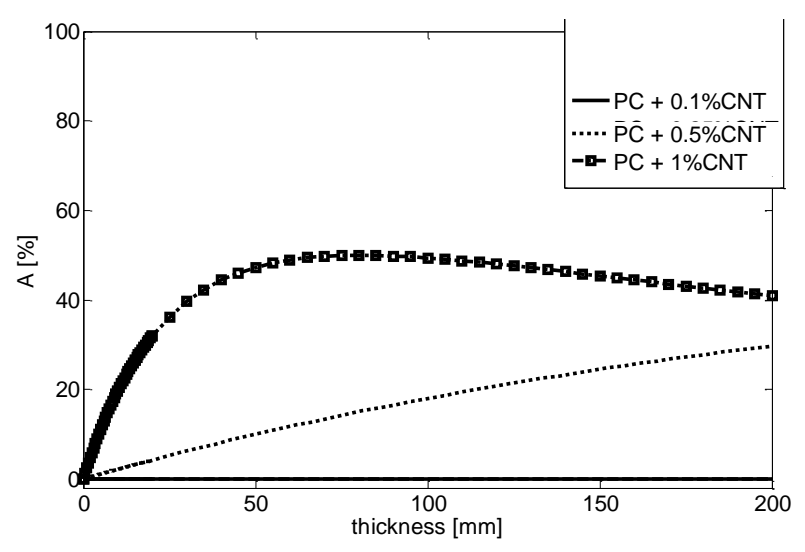

Fig. 11. Absorption in PC samples as a function of thickness.

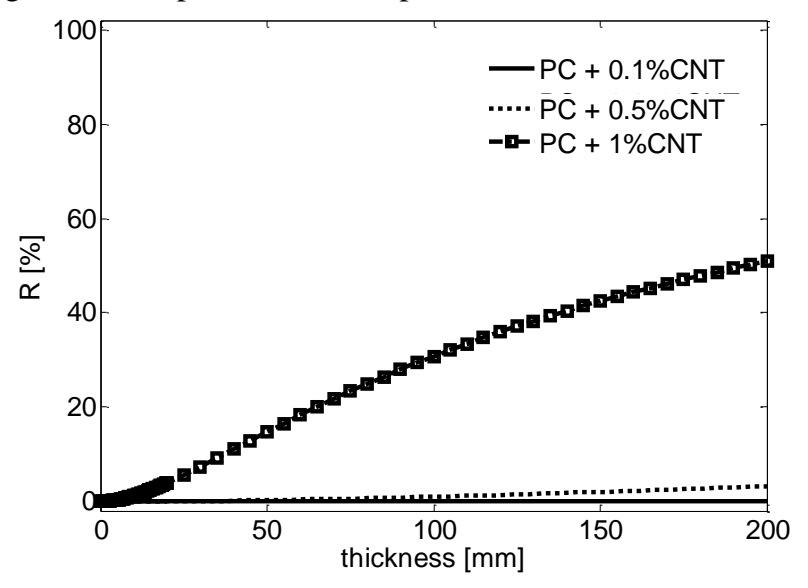

Fig. 12. Reflection in PC samples as a function of thickness. 


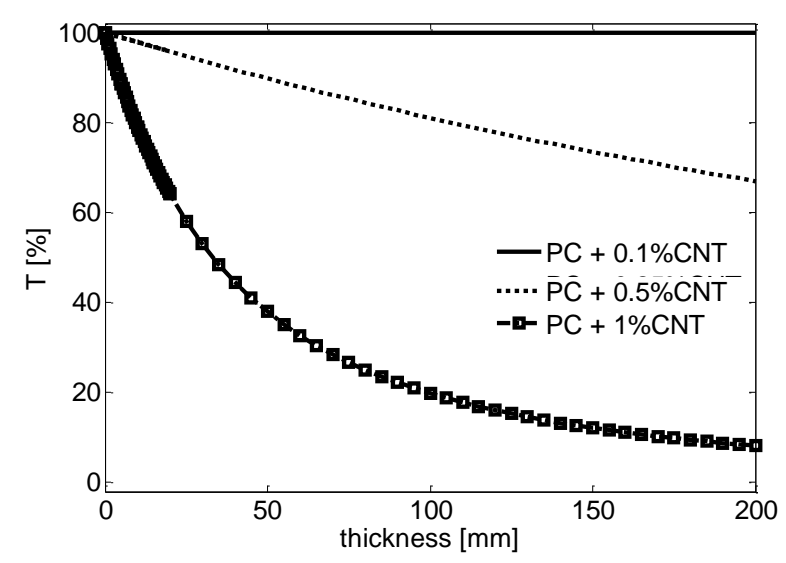

Fig. 13. Transmission in PC samples as a function of thickness.

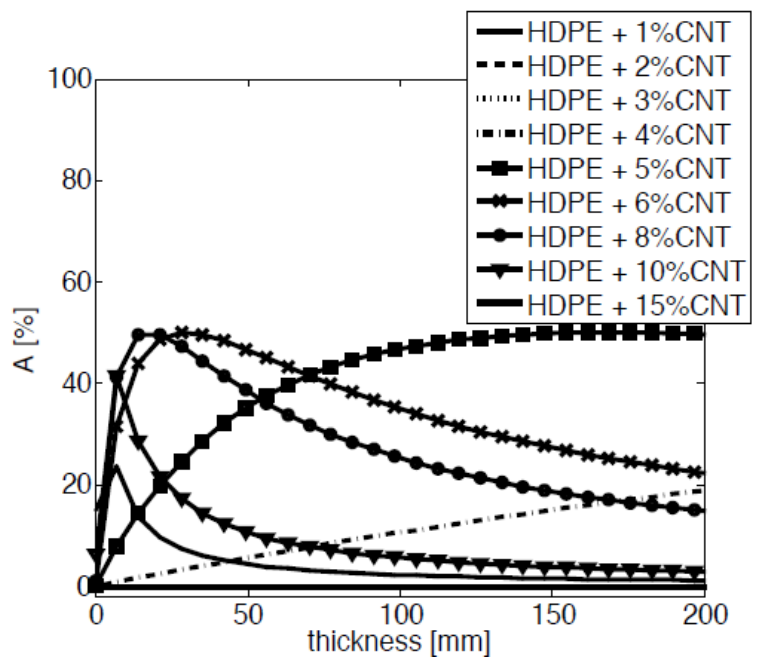

Fig. 14. Absorption in HDPE samples as a function of loading charge of CNT as function of thickness at $1000 \mathrm{~Hz}$.

Absorption is lower than 5\% at low amounts loading for HDPE nanocomposites in Fig. 14. At 4 and $5 \mathrm{wt} \%$ absorption increases towards an intermediate level. Maximum value appears at different thickness and high amount of charge (superior to $5 \mathrm{wt} \%$ ), values growed at thickness lower than $2.5 \mathrm{~mm}$ and progressively decreased at higher thickness. Maximum value appears at different thickness depending on the loading charge; this is due to higher reduction of transmission compared to increase of the reflection. As an example, best absorption of slab with 2 $\mathrm{mm}$ thickness is obtained at $10 \mathrm{wt} \%$ with conductivity around $1 \mathrm{~S} / \mathrm{m}$ and dielectric constant around 1000; while at $20 \mathrm{~cm}$ thickness, best absorption is obtained at $5 \mathrm{wt} \%$ CNT with a conductivity around $0.01 \mathrm{~S} / \mathrm{m}$ and a dielectric constant around 1000. Compared to absorption in GHz range [13,16], the value remains low; but it still exists due to a high conductivity (decreasing the transmission across the sample thickness and a limited increase of dielectric constant (limiting the reflection).

These very nice results suggest a significant absorption at low thickness in highly loaded compounds. The behaviour can be controlled in larger samples by decreasing the charge in particles. In 'semi-transparent' samples, the thickness plays a great role: darker the sample, smaller it has to be in order to absorb the incident signal. If the sample increases in size, the 
reflection will increases and hence reduces the absorption. That sounds really useful at low frequency applications.

\subsection{2 $10 \mathrm{MHz}-40 \mathrm{GHz}$ frequency range}

To confirm our previous discussion, thickness impact on $\mathrm{PC}$ compounds in $\mathrm{GHz}$ frequency range has to be considered and some results compared to other from literature.

For PC samples the lower absorptions are obtained at a thickness of $2 \mathrm{~mm}$ (Fig.15a). The maximum absorption is $80 \%$ at thickness higher than $10 \mathrm{~mm}$ and $40 \mathrm{GHz}$ (Fig. 15c). The samples containing $1 \%$ CNT has a divergent behaviour: the value is low at $700 \mathrm{MHz}$ whatever the thickness, the value linearly increases for $17 \mathrm{GHz}$ but remains lower than the absorption of the others. The samples have an absorption which reaches asymptotic value at high thickness about $80 \%$ at 0.5 and $1 \%$. CNT. These results are in the same range of values than some measured in [3], around $30 \%$ or $60 \%$ absorption in case of CNT gradient loading in PC compounds to control absorption in slab with $2 \mathrm{~mm}$ thickness.

At high frequencies absorption remains constant above a threshold thickness. This means that for this threshold value all the signal penetrating into the slab is totally absorbed.

The fact that absorption does not reach $100 \%$ is due to the part of signal which is reflected at input interface of the slab ( $80 \%$ of absorption corresponds to $20 \%$ of reflection).

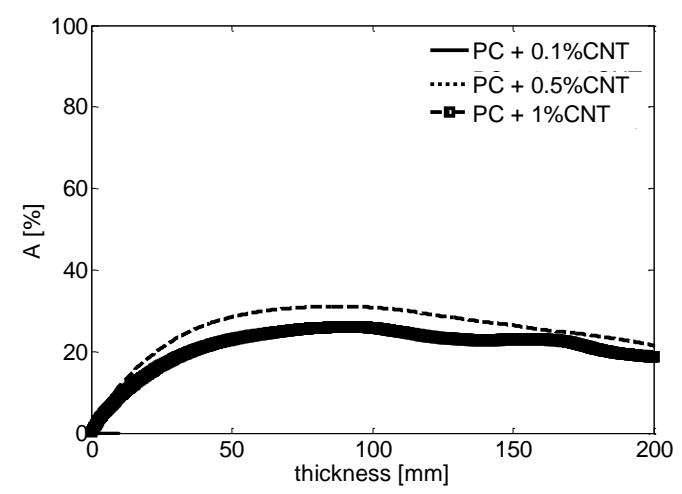

(a) $700 \mathrm{MHz}$

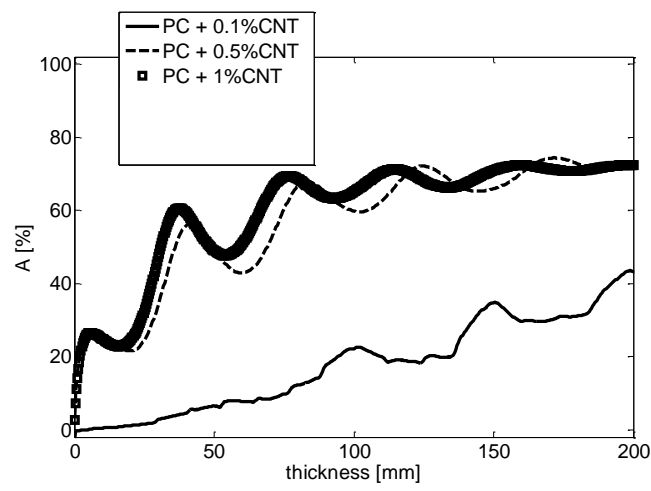

(b) $17 \mathrm{GHz}$ 


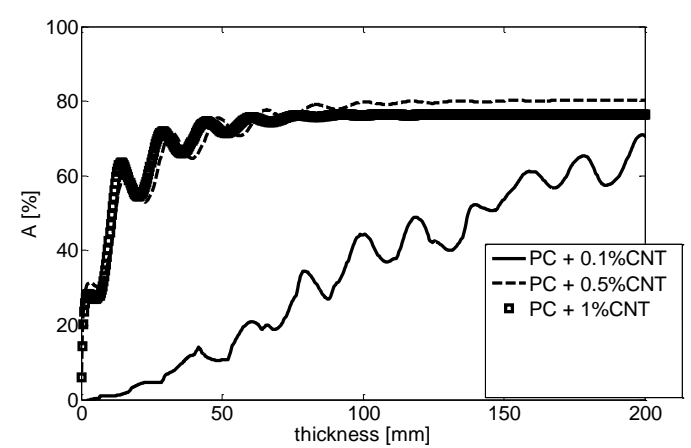

(c) $40 \mathrm{GHz}$

Figure 15 Absorption in PC samples as a function of loading charge of CNT as function of thickness.

\section{CONCLUSION}

Absorption in isotropic nanocomposites loaded by CNT was shown to depend on the CNT fraction and on the thickness. A CNT network leads to an increase of the dielectric constant and the electrical conductivity (especially at frequencies below $1 \mathrm{MHz}$ ). The value was higher for nanocomposites containing entangled CNT network, and the value was lower for composites containing a well-dispersed network. Reflection increased with increasing CNT loading, while transmission decreased. Absorption depends on difference between reflection and transmission at a specific loading charge. If transmission decreases lower than reflection increases, absorption is high e.g. $50 \%$ for $10 \%$ in HDPE. Thickness also impacts the absorption depending on loading charge. Higher the loading, lower the thickness for optimal absorption (up to 50\% by HDPE nanocomposites). Reflection increases at lower thickness for high loading, and hence optimal absorption occurs at lower thickness.

This study is helpful for understanding the mechanisms of absorption in CNT nanocomposites and can be used for the design of FSS absorbers.

\section{ACKNOWLEDGMENT}

Authors thanks the research team of the NANO4WAVES project funded by the Walloon Region through its program "Action de Recherches Concertées".

Authors thank Nicolas Quiévy and Isabel Molenberg for fabricating the PC samples and the Nanocyl company for providing the HDPE samples.

\section{REFERENCES}

[1] Xingcun Colin Tong, "Advanced Materials and Design for Electromagnetic Interference Shielding", CRC Press, November 19, 2008.

[2] Yann. Danlée, Isabelle. Huynen, Christian. Bailly, «"Thin smart multilayer microwave absorber based on hybrid structure of polymer and carbon nanotubes", Applied Physics Letters, vol. 100, no. 21, 2012, pp.213105.1-3.

[3] Yann Danlée, Christian Bailly, Isabelle Huynen, "Thin and flexible multilayer polymer composite structures for effective control of microwave electromagnetic absorption", Composites Science and Technology, vol. 100, August 2014, pp. 182-188.

[4] Jinpil Tak, Yunnan Jin, and Jaehoon Cho, "A dual band metamaterial microwave absorber", Microwave and Optical Technology Letters, vol. 58, no. 99, June 2016, pp. 2052-2055.

[5] Meng Zhang,Tian, Jiang, Yijun Feng, "Design and Measurement Of Microwave Absorbers Comprising Resistive Frequency Selective Surfaces", Journal of Electromagnetic Analysis and Applications, 2014, vol. 6, no. 6, pp. 203-208. 
[6] Rajkumar Jaiswar, Yann Danlée, Henok Mesfin, Arnaud Delcorte, Sophie Hermans, Christian Bailly, Isabelle Huynen, "Absorption Modulation of FSS-Polymer nanocomposites through Incorporation of Graded Conductive nano-fillers", Proceedings of the META 2016 Conference, Malaga, Spain, 25-28 June, 6 pp.

[7]. Rajkumar Rampati Jaiswar, Yann Danlee, Henok Mesfin, Arnaud Delcorte; Sophie Hermans, Christian Bailly, Isabelle Huynen "Absorption Modulation of FSS-Polymer nanocomposites through Incorporation of Graded Conductive nano-fillers" Apllied Physics A, in press,

[8] Narayan Ch Das, Yayong Liu, Kaikun Yang, Weiqun Peng, Spandan Maiti, and Howard Wang. "Single-Walled Carbon Nanotube / Poly ( methyl methacrylate )", Composites for Electromagnetic Interference Shielding. pp. 3-10, 2009.

[9] Yong Li, Changxin Chen, Song Zhang, Yuwei Ni, and Jie Huang. Electrical conductivity and electromagnetic interference shielding characteristics of multiwalled carbon nanotube filled polyacrylate composite films. Applied Surface Science, 254(18), pp. 5766-5771, jul 2008.

[10] Aimad Saib, Lukasz Bednarz, Raphaël Daussin, Christian Bailly, X. Lou, Jean-Michel Thomassin, Christophe Pagnoulle, Christophe Detrembleur, Robert Jerome, and Isabelle Huynen, « Caubon Nanotube Composites for Broadband Microwave Absorbing Materials », IEEE Transactions on Microwave Theory and Techniques (EuMC2005 Special Issue), vol 54, no. 6 (part II), June 2006, pp. 2745-2754.

[11] Nicolas Quiévy, Pierre Bollen, Jean-Michel Thomassin, Christophe Detrembleur, Thomas Pardoen, Christian Bailly and Isabelle Huynen, "Electromagnetic absorption properties of carbon nanotube nanocomposite foam filling honeycomb waveguide structures", IEEE Transactions on Electromagnetic Compatibility (Special Issue on Nanotechnology), vol 54, no.1, February 2012, pp. 43-51.

[12] David Pozar, Microwave engineering, $4^{\text {th }}$ edition, 2012.

[13] Isabelle. Molenberg, "Wideband electrical characterization of carbon-based nanocomposites", Ph.D. Thesis, UCL/EPL June 2011.

[14] Isabelle. Huynen, Nicolas. Quievy, Christian. Bailly, Pierre.Bollen Christophe. Detrembleur, Stéphanie. Eggermont, Isabelle. Molenberg, Jean-Michel Thomassin, Lukasz. Urbanczyk, Thomas. Pardoen, "Multifunctional hybrids for electromagnetic absorption", Acta Materialia, vol. 59, no 8, May 2011, pp 3255-3266.

[15] Isabelle. Huynen, Lukasz. Bednarz, Jean-Michel Thomassin , Christophe. Pagnoulle, Robert. Jerome, Thomas. Pardoen, and Christophe. Detrembleur, "Microwave absorbers based on foamed nanocomposites with graded concentration of carbon nanotubes", Proceedings of the 38th European Microwave Conference, October 2008, pp 5-8.

[16] Yann. Danlée, "Hierarchically organised materials based on polymers and conductive onedimensional nanostructures for the control of electromagnetic propagation", Ph.D. Thesis, UCL/EPL March 2015.

\section{Authors}

Aline Emplit was born in 1987 in Brussels, Belgium. She graduated in Applied Sciences in 2011 from Université catholique de Louvain (U.C.L). She currently follows a PhD degree at Université catholique de Louvain.

Isabelle Huynen was born in 1965 in Brussels, Belgium. She earned the $\mathrm{PhD}$ degree in Applied Sciences in 1994 at the Université catholique de Louvain (U.C.L). Since 1999 she is Research Director of the National Fund of Scientific Research and parttime Professor at UCL.
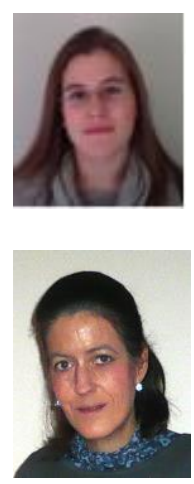